\title{
EXPERIMENTAL AND COMPUTER MODELLING STUDY OF GLOTTAL CLOSING VELOCITY DURING PHONATION
}

\author{
J. Horáček ${ }^{*}$, V. Radolf, V. Bula, P. Šidlof ${ }^{* *}$, A. Geneid ${ }^{* * *}$, A.-M. Laukkanen ${ }^{* * * *}$
}

\begin{abstract}
This preliminary study shows that the impact stress between the colliding vocal folds during phonation should not be evaluated from the maximum velocity of the glottal closing because the velocity of the closing diminishes just before the glottal closure. This phenomenon, which can be caused by a pressure cushion effect in the fast narrowing glottal gap, is demonstrated with measurements from high speed camera images recorded from human and on a physical laboratory model for vowel [u:] phonation and on a threemass computer model employing a Hertz model of impact force. For a more detailed future study of this phenomenon a faster camera has to be used.
\end{abstract}

Keywords: Impact stress, Maximum glottal area declination rate (MADR), High speed imaging.

\section{Introduction}

Periodic mechanical stress is loading the human vocal folds (VFs) due to their collisions during ordinary phonation in chest register where the VFs make full contact and close the glottis. This mechanical stress is the principal reason for VFs tissue damage like nodules or polyps to appear on the VFs' surface which may cause serious voice problems (Titze, 2000). Direct impact (contact) stress measurement has been performed on artificial silicon VF models (see, e.g. Horáček et al., 2018) or on excised canine or human larynges (see e.g. Jiang \& Titze, 1994) and sometimes even in vivo in humans (Verdolini et al., 1999), but it is difficult, unreliable and even in the best case not fully correct, because the pressure sensor between the VFs influences the VFs vibration and the airflow in the glottis.

Another, not direct but noninvasive method to estimate the impact stress (IS) is to study the VFs' vibration by a high speed camera and to determine the closing velocity of the glottis. The maximum velocity of the glottal area when the VFs are approaching each other, the so-called maximum area declination rate (MADR), has been considered as a measure of the IS loading the VFs during collision (Titze, 2006; Titze \& Laukkanen, 2007). Similarly, the maximum velocity of the glottal width during closing has been used for an estimate of IS (Laukkanen et. al. 2018).

The present study compares the velocity of glottal width closing of a male subject's phonation, obtained by high speed imaging, with the results obtained from a similar experiment on a laboratory phonation model and with a computer simulation of VFs' self-oscillation.

\footnotetext{
Ing. Jaromír Horáček, DrSc., Ing. Vojtěch Radolf, Ph.D., Ing. Vítězslav Bula: Institute of Thermomechanics of the Czech Academy of Sciences, Dolejškova 1402/5, 18200 Prague, Czech Republic, jaromirh@it.cas.cz; radolf@it.cas.cz; bula@it.cas.cz

** $\quad$ Assistant professor Ing. Petr Šidlof, Ph.D.: Technical University of Liberec, Studentská 2, 46117 Liberec 1, Czech Republic, petr.sidlof@tul.cz

*** Assistant professor Ahmed Geneid, MD, Ph.D.: Department of Otolaryngology and Phoniatrics - Head and Neck Surgery, Helsinki University Hospital and University of Helsinki, Helsinki, Finland, Box 250 ,00029 HUS; Ahmed.Geneid@hus.fi

${ }^{* * * *}$ Prof. Anne-Maria Laukkanen, Ph.D.: Speech and Voice Research Laboratory, Tampere University, Åkerlundinkatu 5, 33100 Tampere, Finland; Anne-Maria.Laukkanen@tuni.fi
} 


\section{Methods}

First, we present modified results originating from our previous experimental study of human phonation characteristics during the voice therapy method using phonation into tubes (Laukkanen et. al. 2018). High-speed laryngoscopic data for human VFs' vibration during phonation of a neutral vowel between [o:] and [u:] at a comfortable speaking pitch and loudness were collected using a Kay Pentax Color HighSpeed Video System 9710 with a resolution of 512x512 pixels. The sampling frequency was 2000 frames/second. The glottal width variation GW(t) values (in pixels) were recalculated to dimensionless quantities by dividing them by the vocal fold width (in pixels). Then the time derivative of the glottal width variation $(\mathrm{dGW}(\mathrm{t}) / \mathrm{dt})$, representing the speed of the glottal opening and closing phases, was in the present paper calculated by the method of backward differences.

Second, an "in vitro experiment" was performed on an artificial model of phonation for vowel [u:] using the mean airflow rates $\mathrm{Q}=0.08-0.25 \mathrm{~L} / \mathrm{s}$, the mean subglottic pressures $\mathrm{Psub}=1.45-2.24 \mathrm{kPa}$ and the fundamental phonation frequencies of the VFs' self-oscillations in the range $\mathrm{F} 0=110-113 \mathrm{~Hz}$, i.e. within physiologically relevant values for male voice production. The air was flowing from a model of the lungs to a trachea model and then through a 1:1 scaled three layer VFs' model to a vocal tract model, see details in (Horáček et al., 2017). A high-speed CCD camera NanoSense MkIII (maximum resolution 1280x1024 pixels) with a camera zoom lens Nikon AF micro Nikkor $60 \mathrm{~mm}$ was included in the measurement set up for the analyses of the VFs' vibration. The rate of 3000 frames/second was used.

Third, the results of the in vivo and in vitro measurements of the glottal closing speed were compared with the values predicted by numerical simulations using an aeroelastic model of VFs' self-oscillations (Horáček et al., 2005). The three-mass VFs model is based on the equations of motion for the two-degreeof-freedom dynamic system using the potential theory for airflow in glottis and the Hertz impact theory for the VFs' collision (Brepta \& Prokopec, 1972; Goldsmith, 1960). We can note that according to the Hertz theory the maximum contact (impact) stress IS during the VFs' collision is approximately proportional to the velocity V0 of glottal closing just before the complete closure in line with the formula:

$$
I S \approx \text { const. } V_{0}^{2 / 5} \text {, }
$$

where the constant is given by the material properties and the curvature of impacting surfaces. The Hertz theory was used by Horáček et al. (2005) in computer modelling of VFs' self-sustained vibration for estimation of impact stress between the colliding VFs, and the results were found to be in good agreement with measurements on the artificial VFs (Horáček at al., 2016) as well as with results on excised larynges (Horáček at al., 2009). Such good agreement is probably caused by high damping of the generated deformation waves in the VF tissue, which impedes reflections of the waves.

\section{Results}

Figure 1 shows the results from the measurement of phonation characteristics in the human subject for seven cycles of the VFs' vibration. Following the first three cycles it can be detected that the maximum velocity of the glottis closing, given by the absolute value of the time derivative of the glottal width (dGW(t)/dt), is followed by lower values of glottal width decreasing velocity $V_{0}$ just before the glottis closure, which according to eq. (1) implicates lower impact stress IS. The markedly visible decrease of the velocity magnitude is substantially influenced by the too low sampling frequency given by the high speed camera used. In the first cycle, after the maximum velocity of the glottal closing (MADR), the velocity $V_{0}$ decreases by ca $40 \%$ before the collision, in the second cycle it decreases by about $70 \%$ and in the third cycle by ca $90 \%$. This indicates that the glottal closing velocity rapidly decreases just before the closure. The results shown in Fig.1 are influenced not only by a low sampling frequency but also naturally by jitter and shimmer known as normal features in human phonation destroying the perfect periodicity of the measured signals.

Similar results are shown in Fig.2 for measurement on the physical model of phonation where the maximum velocity of the glottal area $(G A)$ closing (MADR) is followed by lower velocity values of the glottal gap in all oscillation cycles. For example, the minimum derivative of the glottal area $\mathrm{d} G A(t) / \mathrm{dt} \cong 5000 \mathrm{~mm}^{2} / \mathrm{s}$ (i.e. MADR $\cong 5000 \mathrm{~mm}^{2} / \mathrm{s}$ ) appears in the seventh cycle, however a much lower velocity $V_{0} \cong 300 \mathrm{~mm}^{2} / \mathrm{s}$ occurs at the time instant just before the contact of the VFs at the time instant, where $G A=0$ and $d G A(t) / d t=0$. This effect is visible here better than in Fig. 1 due to the higher filming frame rate used in this experiment. 


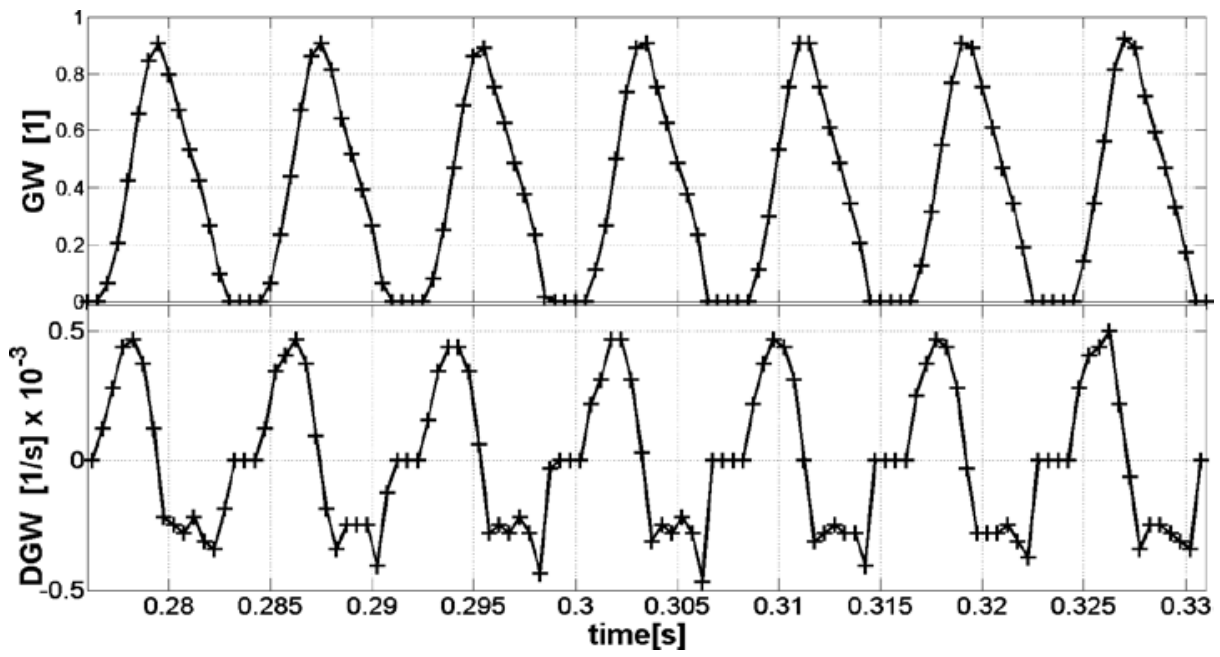

Fig. 1: Glottal width variation waveform $G W(t)$ and its derivative $D G W(t)$, obtained from high-speed images for vowel phonation - measurement on human subject (sustained vowel between [u:] and [o:], $P_{\text {sub }} \cong 2.03 \mathrm{kPa}, F_{0}=126 \mathrm{~Hz}$, sampling frequency $2 \mathrm{kHz}$ ).

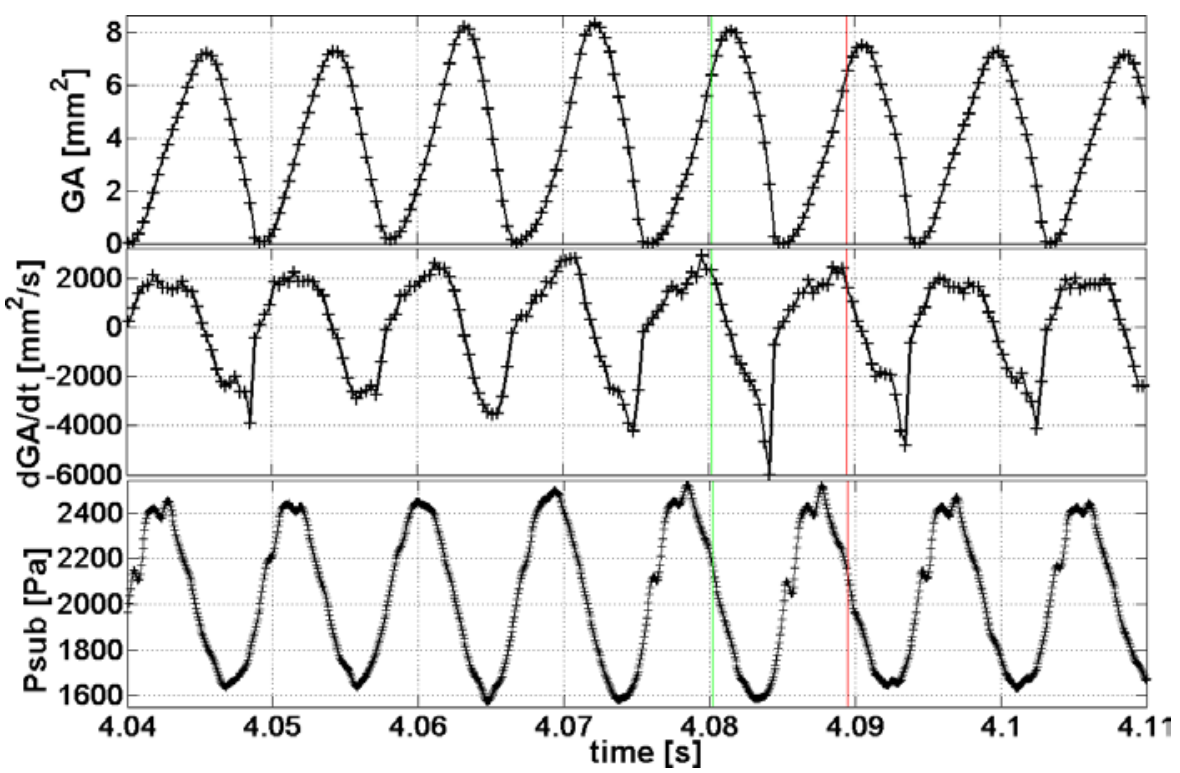

Fig. 2: Glottal area variation waveform (top) and its derivative (bottom), obtained from high-speed images for vowel phonation - measurement on the experimental model $\left(Q=0.20 \mathrm{~L} / \mathrm{s}, P_{\text {sub }}=2.03\right.$ $\mathrm{kPa}, \mathrm{F}_{0}=111 \mathrm{~Hz}$, sampling frequency $3 \mathrm{kHz}$ ).

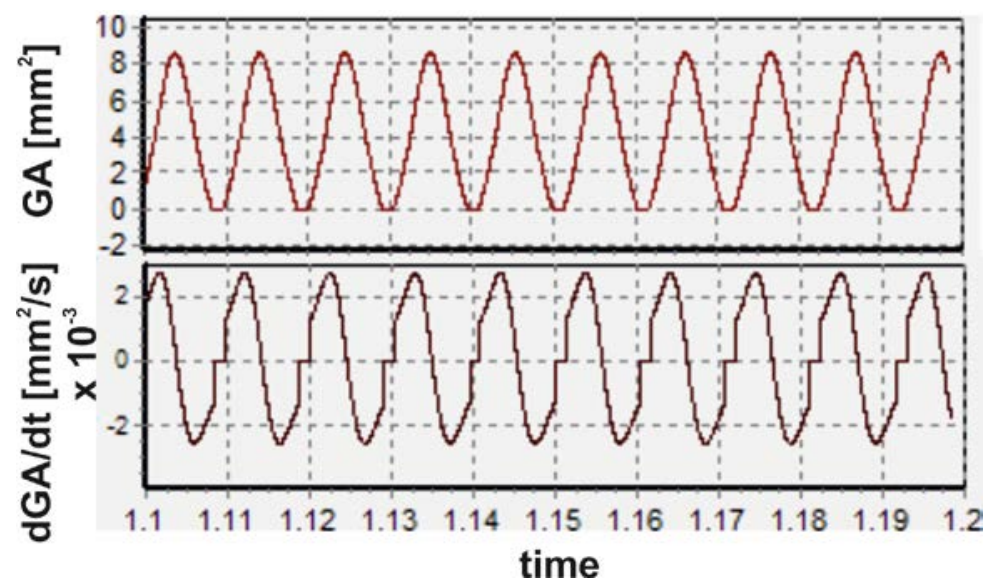

Fig. 3: Glottal area variation waveform (top) and its derivative (bottom), obtained from the computer simulation $\left(Q=0.12 \mathrm{~L} / \mathrm{s}, P_{\text {sub }}=180 \mathrm{~Pa}, F_{0}=96 \mathrm{~Hz}\right.$, sampling frequency $\left.50 \mathrm{kHz}\right)$. 
Figure 3 obtained by the numerical simulation of the VFs oscillation without any vocal tract shows also a braking effect during the glottis closing phase. After MADR $\cong 2500 \mathrm{~mm} 2 / \mathrm{s}$ the glottal gap velocity decreases to the impact velocity $\mathrm{V} 0 \cong 1500 \mathrm{~mm} / \mathrm{s}$ just before the glottis closure. The waveforms in Fig. 3 are computed for lower subglottal pressure Psub compared to the examples of the measured results shown in Figs. 1 and 2. For the higher Psub values comparable with the experiments no braking effect was visible in the glottal width diminishing before the glottis closure in the numerically simulated waveforms. It is probably caused by the fact that no reactance of the vocal tract and inviscid flow were considered in the computer model.

\section{Conclusions}

The maximum glottal area time derivative during the glottal closure (so-called maximum area declination rate; MADR) is considered a measure of the maximum impact stress between the colliding VFs. However, following the graph for the derivative of the glottal width variation it was found in the present study, that in reality the glottal closing speed is noticeably reduced just before the VFs' contact. Thus, MADR is not an adequate measure of impact stress, whereas the glottal area derivative just prior to glottal closing is. The glottal closing speed should be investigated in detail in a future study using higher frame rates in high speed imaging.

\section{Acknowledgement}

The study was supported by a grant from the Czech Science Foundation: No. 19-04477S "Modelling and measurements of fluid-structure-acoustic interactions in biomechanics of human voice production."

\section{References}

Brepta, R. and Prokopec, M. (1972) Wave propagation and impacts in solids. Praha: Academia (in Czech).

Goldsmith, W. (1960) Impact. London, UK: Arnold Ltd.

Horáček, J., Šidlof, P. and Švec, J. G. (2005) Numerical simulation of self-oscillations of human vocal folds with Hertz model of impact forces. Journal of Fluids and Structures, 20, pp 853-869.

Horáček, J., Laukkanen, A.M., Šidlof, P., Murphy, P. and Švec, JG. (2009) Comparison of acceleration and impact stress as possible loading factors in phonation. A computer modeling study. Folia Phoniatrica et Logopaedica, 61, 3, pp 137-145.

Horáček, J., Bula, V., Radolf, V. and Šidlof, P. (2016) Impact stress in a self-oscillating model of human vocal folds. Journal of Vibration Engineering \& Technologies 4, 3, pp 183-190.

Horáček, J., Radolf, V., Bula, V. and Košina, J. (2017) Experimental modelling of phonation using artificial models of human vocal folds and vocal tracts. In: Engineering Mechanics 2017 (Fuis, V. ed.). Brno: University of Technology, pp. 352-385.

Horáček, J., Radolf, V. and Laukkanen, A-M. (2018) Impact stress in water resistance voice therapy: A physical modeling study, Journal of Voice, In Press, Available online 5 June 2018, https://doi.org/10.1016/j.jvoice.2018.01.025.

Jiang, J. and Titze, I. (1994) Measurement of vocal fold intraglottal stress and impact stress. Journal of Voice, 8, pp 132-144.

Laukkanen, A.M., Geneid, A., Bula, V., Radolf, V., Horáček, J., Ikavalko, T., Kukkonen, T., Kankare, E. and Tyrmi, J. (2018) How much loading does water resistance voice therapy impose on the vocal folds? An experimental human study. Journal of Voice, In Press, Available online 22 November 2018, https://doi.org/10.1016/j.jvoice.2018.10.011.

Titze, I.R. (2000) Principles of voice production. (2nd printing), Iowa City, IA: National Center for Voice and Speech.

Titze, I.R. (2006) Theoretical analysis of maximum flow declination rate versus maximum area declination rate in phonation. Journal of Speech, Language, and Hearing Research, 49, pp 439-447.

Titze, I.R. and Laukkanen, A.M. (2007) Can vocal economy in phonation be increased with an artificially lengthened vocal tract? A computer modeling study. Logopedics Phoniatrics Vocology, 32, 4, pp 147-156.

Verdolini, K., Hess, M.M., Titze, I.R., Bierhals, W. and Gross, M. (1999) Investigation of vocal fold impact stress in human subjects. Journal of Voice, 13, pp 184-202. 